\title{
Assessment of Educational Needs for Common Diseases to Promote the Skills of Medical Students in Designing the Med ED Application
}

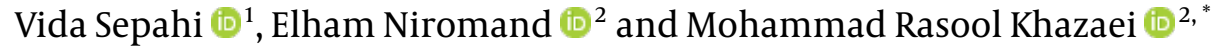 \\ ${ }^{1}$ Education Development Center, Kermanshah University of Medical Sciences, Kermanshah, Iran \\ ${ }^{2}$ Fertility and Infertility Research Center, Health Technology Institute, Kermanshah University of Medical Sciences, Kermanshah, Iran \\ "Corresponding author: Fertility and Infertility Research Center, Health Technology Institute School of Medicine, University Ave, Shahid Shiroudi Blvd, Zip-code: 67148-69914, \\ Kermanshah, Iran. Tel/Fax: +98-8334281563, Email: mrasoolkhazaei@gmail.com \\ Received 2021 December 11; Accepted 2021 December 15.
}

Keywords: Educational Needs Assessment, Medical Students, Common Diseases

\section{Dear Editor,}

Rapid scientific advancement and the need for selecting optimal and effective concepts and content highlight the importance of attention to the process of educational planning based on fundamental needs. Apart from the opponents and proponents of needs assessment, it should be borne in mind that needs assessment is essential to educational planning, and goals and directions could not be determined without such assessments. This is because needs assessment is essential to providing the required information for educational curricula, evaluations, holding educational organizations and institutions accountable, adaptation to change, identifying weaknesses and basic requirements in curricula, and accomplishing participatory endeavors $(1,2)$. Undoubtedly, general practitioners are key members of the healthcare team and play a pivotal role in the management and treatment of outpatients. Internship and apprenticeship courses are essential to the adoption of critical skills and professional capabilities in medical students, constituting $50 \%$ of medical curricula as an indispensable means of preparing medical students for their future careers (3). In line with global health policies, guidelines of the World Health Organization (WHO), and policymaking for the transformation of the national health sector, the empowerment of general practitioners as the frontline of patient treatment is highly prioritized, and this goal could only be achieved by promoting proper education simultaneous with academic courses and constant virtual training. Therefore, we aimed to design a mobile application for common diseases to enhance the education and skills of medical students. Prior to developing the application, a needs assessment was performed to provide the required educational content.

The current survey was a needs assessment conducted by the Delphi method in three stages. Due to the COVID19 pandemic, students could not attend the survey at a specific time and place; therefore, we opted for a survey design. In total, 10 faculty members of various module departments and 30 medical stagers and interns were selected as the target population via purposive sampling from Kermanshah University of Medical Sciences, Iran. The participants had adequate experience and full-time attendance in various educational and clinical fields, which enabled them to determine the educational needs of various departments. At the first stage, faculty members were asked to prepare a form containing the diseases and complications (signs and symptoms) that must be identified by medical interns and could also be presented virtually (i.e., in the form of a software application) without prioritization (minimum of three and maximum of seven items). At the second stage, the form prepared by the faculty members and experts was sent to the students, and they were asked to select the items with the highest priority. At the third and final stage, the collected data were recorded in the third form, and a list of the given comments by the students was provided to the faculty members to provide feedback and identify the strengths and weaknesses. A grading system was used for prioritization, and the participants were asked to determine their educational priorities in a ballot and comment on the feedback of the faculty members as well. Scores one and five were assigned to the first and fifth priorities, respectively, and the priorities were de- 
cided based on the obtained score in each educational department. The items with the highest score achieved the highest priority, followed by the lower scores (lower priorities). Via consensus with five experts (faculty members of module departments, educational developers, and media developers), the researchers selected items 1-20, which had the highest priority, and the remaining items were eliminated.

As a result, 47 items were considered to be the foremost educational needs of medical students by 10 experts. After integration and the elimination of similar items, 40 items remained in the list, which was delivered to 30 students in a separate form to select 20 items. After the students completed the form, 20 items that were repeated most were selected as the main priorities of medical education. Via consensus and considering financial restrictions, the researchers selected 15 items as the electronic content for the development of the software application. These items included: COVID-19, water and electrolyte disorders, hypovolemic shock, approach to dehydration, sinusitis, asthma and dyspnea, neonatal icter, allergic rhinitis, pneumonia and respiratory infections, diabetes symptoms and diagnosis, management and treatment of type I diabetes, management and treatment of type II diabetes, cardiac angina, approach to chest pain, approach to MI.

Several studies have been focused on needs assessment using various methodologies. Yamani et al. (4) performed the needs assessment of the MSc nursing curriculum in 2010 - 2011 using the Delphi method and expert opinions (nursing experts) similar to the current research. In the first stage of the Delphi method, 119 tasks were extracted. At the second stage, 36 essential tasks were selected by a focus group (4). On the other hand, Mirzaeikarzan et al. (5) performed the needs assessment of the faculty members of Ilam University of Medical Sciences (Iran) using Kaufman's three-dimensional needs assessment model in two main phases. Based on the mentioned model, the researchers assessed the needs of the participants from the perspective of system management, faculty members, and students. In the first phase of the research, purposive sampling was used to select authorities and students to conduct semistructured interviews (5).

Various methods and models could be used for needs assessment, which are selected depending on the type of the studies in this regard. In the current research, we used the Delphi method and comments of the faculty members to extract the educational needs of the students for the main project (i.e., software application), part of which involved needs assessment. Finally, the Med ED software ap- plication was designed and developed based on the needs assessment.

\section{Footnotes}

Authors' Contribution: MRK designed and prepared the initial draft of the article. EN collected information. VS prepared and finalized the article.

Conflict of Interests: There is no conflict of interest. Funding/Support: This project was funded by the National Agency for Strategic Research in Medical Education, Tehran, Iran; grant No. was 993459.

\section{References}

1. Toulabi T, Sheikhian A, Galehdar N, Anbari K, Mohammadi E, Majidimehr M. Educational needs of faculty members of Lorestan University of Medical Sciences by Delphi Technique. J Nurs Educ. 2015;4(2):28-37.

2. Norman GR, Shannon SI, Marrin ML. The need for needs assessment in continuing medical education. BMJ. 2004;328(7446):9991001. doi: 10.1136/bmj.328.7446.999. [PubMed: 15105326]. [PubMed Central: PMC404504].

3. Midgley K. Pre-registration student nurses perception of the hospitallearning environment during clinical placements. Nurse Educ Today. 2006;26(4):338-45. doi: 10.1016/j.nedt.2005.10.015. [PubMed: 16406618].

4. Yamani N, Alizadeh M, Changiz T, Taleghani F. Need Assessment for Master of Science in Family Nursing Curriculum: Using Delphi Technique. Iran J Nurs. 2012;24(74).

5. Mirzaeikarzan A, Keikhavani S, Hosseinzade M. Educational needs assessment of faculty members in Ilam UMS. J Med Educ Develop. 2013;6(11):61-71. 